This is a postprint version of the following published document:

Pinzon, P.J.; Pérez, I.; Vazquez, C. Efficient Multiplexer/Demultiplexer for Visible WDM Transmission over SI-POF Technology. IEEE Journal of Lightwave Technology 37(2015) 17, September, pp. 3711-3718. Available in http://www.dx.doi.org/10.1109/JLT.2015.2455335

(C) 2015. IEEE. Personal use of this material is permitted. Permission from IEEE must be obtained for all other uses, in any current or future media, including reprinting/republishing this material for advertising or promotional purposes, creating new collective works, for resale or redistribution to servers or lists, or reuse of any copyrighted component of this work in other works. 


\title{
Efficient Multiplexer/Demultiplexer for Visible WDM Transmission over SI-POF Technology
}

\author{
Plinio Jesús Pinzón, Isabel Pérez Garcilópez, and Carmen Vázquez
}

\begin{abstract}
A five channel step index plastic optical fiber proposal for a multiplexer/demultiplexer having insertion losses $(I L)$ of 2.9-4 dB, pass bandwidths at $-3 \mathrm{~d} \mathrm{~B}>30$ $\mathrm{nm}$, crosstalk attenuation $>30 \mathrm{~dB}$ and size of $\sim 65 \mathrm{~mm} \times$ $55 \mathrm{~mm}$, is demonstrated. It is based on a reflective diffraction grating with blazed profile and an aspheric lens. The theoretical analysis presented is used to further reduce the system size to $\sim 37 \mathrm{~mm} \times 30 \mathrm{~mm}$ and to increase the number of channels to 8 keeping $I L s<4.5 \mathrm{~dB}$. Experimental results have good agreement with theoretical expectations.
\end{abstract}

KeyWords: Demultiplexer, diffraction grating, multiplexer, polymer optical fiber, visible, wavelength division multiplexing.

\section{Introduction}

Today the volume of data transmitted by short-range networks, especially by In-Home networks, both to the Internet Service Provider and between different terminals, is increasing beyond the Gbit/s, exceeding the capabilities of current networking technologies [1]. This is due to the fast growth of new multimedia services like IPTV, multiroom/multivision configurations, high-definition TV or remote "face-to-face communication," among others [2]. On the other hand, polymer optical fiber (POF) has been proposed, and recognized, as one of the most promising transmission media for implementing high speed and low cost short-range communication networks [1]. Specifically, in scenarios, such as local area networks, inhome, and office networks [3], as well as in automotive [4] and avionic multimedia buses, or in data center interconnections [5]. Among the different POF types, the step index POF (SIPOF) with a $980 \mu \mathrm{m}$ core diameter of polymethyl-methacrylate (PMMA) and numerical aperture (NA) of $\sim 0.5$ offers several advantages, especially in the short-range scenarios listed previously, due to its potential low cost for its easiness of handling, installation, splicing and connecting. However, SI-POF suffers from high modal dispersion due to its large $N A$, limiting its bandwidth distance product to $\sim 50 \mathrm{MHz} \times 100 \mathrm{~m}$ [6], and providing acceptable attenuation only in the visible spectrum (VIS) $(\sim 0.17 \mathrm{~dB} / \mathrm{m}$ at $650 \mathrm{~nm})[7]$.

$\mathrm{Gbit} / \mathrm{s}$ transmission capacity of SI-POF links has been widely demonstrated in recent years, using single channel $(c h)$ based

This work was supported by the Spanish Ministry of Economy and by Madrid region under Grants TEC2012-37983-C03-02 and P2013/MIT-2790.

The authors are with the Electronics Technology Department, Universidad Carlos III de Madrid, Madrid 28911, Spain (e-mail: ppinzon@ing.uc3m.es; isaper@ing.uc3m.es; cvazquez@ing.uc3m.es). systems (typically at $650 \mathrm{~nm}$ ) with different advanced modulation formats and/or adaptive electrical equalization techniques. Reported simulations shows that data rates of 1.25, 2.1 [8] and even $6.2 \mathrm{Gbit} / \mathrm{s}$ [9], via up to $50 \mathrm{~m}$, can be reached using a single $c h$, with NRZ, CAP-64 and QAM512 modulations, respectively, with LED [8] and LD [9] transmitters (Txs). More recent experimental systems demonstrate data rates of MultiGbit/s up to $50 \mathrm{~m}$ with LD Txs [6] and up to $3 \mathrm{Gbit} / \mathrm{s}$ over $25 \mathrm{~m}$ using 8-PAM with LED Txs [10]. Fully integrated systems that offer real-time SI-POF links at $1 \mathrm{Gbit} / \mathrm{s}$ via up to $50 \mathrm{~m}$ using $M$ PAM modulation with a LED $T x$ have also been reported [11]. But SI-POF's data transmission capacity needs a greater exploitation to meet user requirements for higher data rates with a low power consumption and low cost. This generates an interest in the development of new systems for Multi-Gbit/s transmission over SI-POF, including new transmitters and receivers, and in new standards, based on SI-POF [1], [4]. After exploiting the capabilities of a single $c h$ transmission, visible wavelength $(\lambda)$ division multiplexing (visible WDM) is proposed as a solution to expand the capacity of SI-POF based systems. Current proposals of visible WDM transmission over SI-POF are based on spectral grids with chs between 400 and $700 \mathrm{~nm}$ [12], using LDs. Visible WDM systems using offline-processed DMT modulation, and data rates up to 14.77 [13] and $21 \mathrm{Gbit} / \mathrm{s}$ [5] over $50 \mathrm{~m}$, with 4 and $6 \mathrm{chs}$, respectively, have been recently reported. In both systems, the average data rate per $c h$ is about $3.5 \mathrm{Gbit} / \mathrm{s}$ with bit error rate of $1 \times 10^{-3}$.

In WDM systems two key-elements have to be introduced: a multiplexer (Mux) and a demultiplexer (DeMux). On the other hand, a limitation of visible WDM links over SI-POF is the power penalty due to the current Muxes/DeMuxes high insertion losses (ILs), limiting the transmission capacity of each $c h$ in comparison with single $c h$ systems, for the same transmitted power [9]. This can be overcome by reducing the Muxes/DeMuxes' ILs, and/or increasing the transmission power. But the latter solution increases the system power consumption and is only suitable for scenarios where working outside the eye-safety-limit is allowed [5], [13]. Mux/DeMux devices with $I L s \approx 3.5 \mathrm{~dB}$ per $c h$ allow to establish SI-POF visible WDM systems with transmission power per $c h$ near to the eye safety limit $(<1 \mathrm{~mW})$, that can be used in network topologies [14], where the fiber containing all the WDM chs is kept away from the end user. Therefore, the development of Mux/DeMux devices with low ILs and higher ch counts than the current proposals is essential in order to implement efficient visible WDM SI-POF links, working near the eye-safe-limit and using low-power technology [9], especially for in-home and office networks [14]. On the other hand, the design of Mux/DeMux 
TABLE I

CHARACTERISTICS OF SOME MULTIPLEXER/DEMULTIPLEXER DEVICES FOR SI-POF WDM SYSTEMS

\begin{tabular}{|c|c|c|c|c|c|c|c|}
\hline Technology & \#Elements & Size $[\mathrm{mm} \times \mathrm{mm}]$ & Channels [nm] & $I L[\mathrm{~dB}]$ & $C T A[\mathrm{~dB}]$ & [Ref.], Year & Remarks \\
\hline Planar blazed diffraction grating (600 lines/mm) & 2 & (1) & 520,650 & 6.2 to 7.5 & 25 & [19], 2002 & (2) \\
\hline Thin film filters & $>4^{(1)}$ & (1) & 520,655 & 3 to 5 & 20 & [19], 2002 & (2) \\
\hline Holographic concave grating reflector (1200 lines/mm) & 1 & $\sim 20 \times 35^{(1)}$ & $520,570,655$ & 2 & 20 & [21], 2005 & (3), (4), (5) \\
\hline Ellipsoid and Spherical grating mirrors (1200 lines/mm) & 1 & $\sim 30 \times 26^{(1)}$ & 480,520630 & (1) & 30 & [23], 2008 & (3) (4) \\
\hline Prism & 2 & $79 \times 94$ & $470,520,655$ & 12 to 19 & 4.6 to 6.8 & [20], 2008 & (2) \\
\hline Blazed grating on an aspheric mirror (500 lines $/ \mathrm{mm}$ ) & 1 & $13 \times 20$ & $450,520650^{(7)}$ & (1) & (1) & [22], 2013 & (3) (5) (6) \\
\hline Thin film filters & 12 & $\operatorname{Large}^{(1)}$ & $405,450,528,646$ & 3.3 to 5.7 & $>30$ & [13], 2014 & (2) (8) \\
\hline Planar Holographic Diffraction Grating (1800 lines/mm) & 2 & diam. $\geq 75^{(1)}$ & $405,442,459,490,515,655$ & $<10$ & 20 to $>30$ & [5], 2014 & (2) \\
\hline \multirow[t]{2}{*}{ Planar blazed diffraction grating (600 lines/mm) } & 2 & $65 \times 55$ & $504,515,650$ & 3.5 to 4.5 & $>30$ & [24], 2014 & (2) \\
\hline & & & $405,470,530,588,650$ & 2.8 to 3.9 & $>30$ & In this work & (2) \\
\hline Planar blazed diffraction grating (1200 lines $/ \mathrm{mm}$ ) & 2 & $57 \times 50$ & $435,465,497,530,562,595,625,655$ & 3.2 to 4.5 & $>30$ & In this work & (2) \\
\hline
\end{tabular}

Notes: (1) Unspecified or not analyzed; (2) Experimental results with ports made of SI-POF; (3) Simulations without experimental results; (4) The diameter of the SI-POF is not considered in the simulations; (5) A detection layer is considered at the output; (6) SI-POF input with $0.38 \mathrm{NA}$ and $0.98 \mathrm{~mm}$ diameter; (7) An extra channel at 405nm is included, but only chs with high CTA are considered; (8) There is a thin-film filter in each output to improve the crosstalk attenuation.
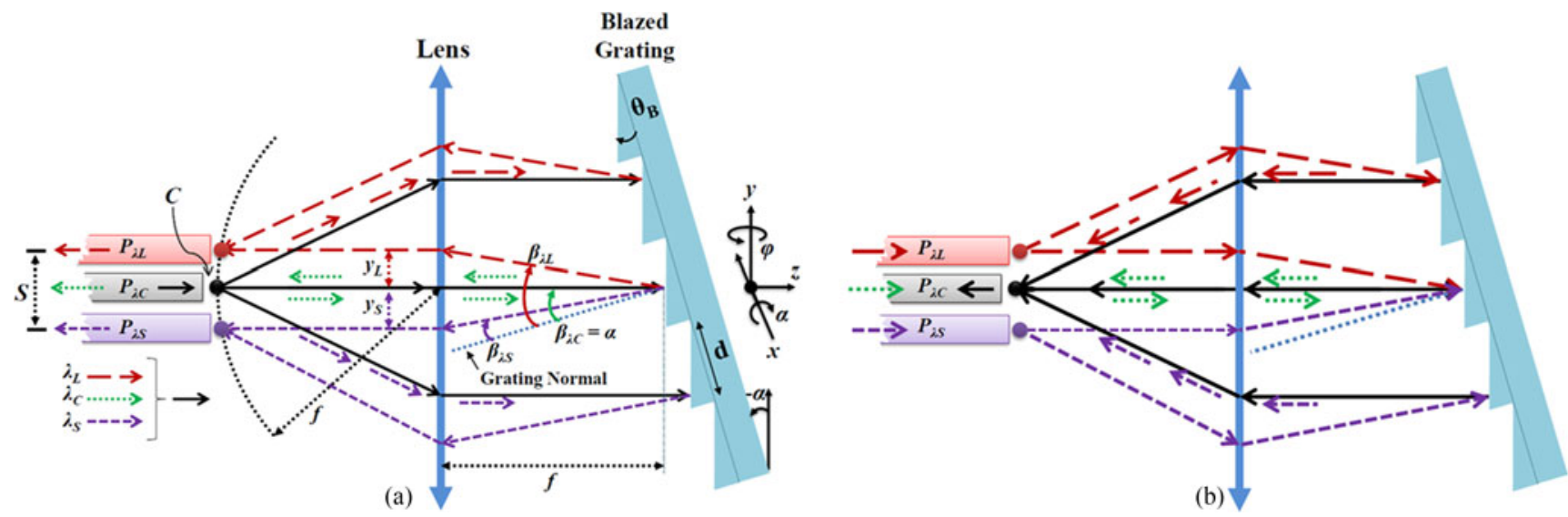

Fig. 1. Layout of SI-POF Mux/DeMux: (a) ray tracing in demultiplexing and (b) ray tracing in multiplexing.

devices with a reduced size is another important requirement. In this paper, a five $c h$ diffraction grating based Mux/DeMux for visible WDM transmission over SI-POF technology, with low $I L$, large rejection bandwidth, and a compact size, in comparison with the current state-of-the-art, is presented. Thus, we first review the Mux/DeMux devices for visible WDM based SI-POF networks. Then, we analyze the most important requirements imposed by the SI-POF characteristics in the design of diffraction grating based Muxes/DeMuxes. We next described the five ch Mux/DeMux design and its experimental characterization. Finally, we present the discussion and the relevant conclusions.

\section{Muxes/DeMuxes for visible WDM over SI-POFS}

Muxes/DeMuxes are basic elements in the WDM approach. However, most Muxes/DeMuxes for glass optical fibers (GOFs) [15]-[17], or for graded index POF with low NA [18], are not suitable for SI-POFs. This is due to the spectral range and spectral bandwidths used for visible WDM over SI-POFs [12], and the SI-POF physical characteristics. A summary of different Muxes/DeMuxes for SI-POF is shown on Table I. They are mainly based on thin-film filters [13], [19], prisms [20], and diffraction gratings [5], [21]-[23]. Muxes/DeMuxes based on planar blazed diffractions grating are to date a promising option for implementing compact devices with low ILs and multiple chs. The next lowest $I L<4.5 \mathrm{~dB}$ is achieved in a diffraction grating based Mux/DeMux of three chs [24]. The maximum number of chs is reported in [5], but the ILs are up to $10 \mathrm{~dB}$. In this paper, we report a five $c h$ Mux/DeMux with $I L s<4 \mathrm{~dB}$ and an eight $c h$ Mux/DeMux with ILs $<4.5 \mathrm{~dB}$, both with crosstalk attenuation $(C T A)>30 \mathrm{~dB}$.

\section{Theoretical basic and proposed scheme}

Fig. 1 shows the layout of the proposed SI-POF Mux/DeMux. It consist of a reflective blazed diffraction grating with blazing angle $\theta_{B}$, grating pitch $d$ and tilt angle about the $x$-axis $\alpha$; and a collimating/focusing lens with effective focal length $f$ and pupil diameter $D_{L}$. The light, which emerges [see Fig. 1(a)] from the common port, $P_{\lambda C}$, located at $C$ point, contains the central wavelength $\lambda_{C}$, and the wavelengths of the extreme $c h s$ to be demultiplexed, which are referred as $\lambda_{S}$, for the shorter $\lambda$ (at $\sim 400 \mathrm{~nm}$ ) and as $\lambda_{L}$, for the longer $\lambda$ (at $\sim 700 \mathrm{~nm}$ ). The system is bidirectional. So the rays that emerge from $P_{\lambda S}$ and $P_{\lambda L}$ are multiplexed in $P_{\lambda C}$; see Fig. 1(b).

A light beam is directed at the diffraction grating. The grating reflects back a plurality of collimated beams of light, each within a different $\lambda$ range and at a specific direction defined by 


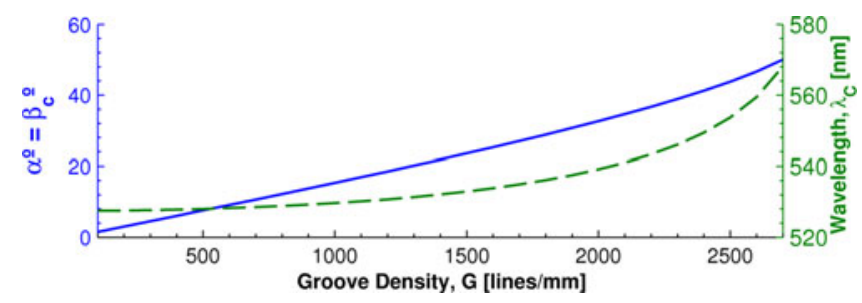

Fig. 2. Dependence of $\lambda_{C}$ (dashed line, right axis) and the $\alpha=\beta_{\lambda C}$ (solid line, left axis) with the groove density for $\lambda_{S}=405 \mathrm{~nm}$ and $\lambda_{L}=650 \mathrm{~nm}$.

a diffraction angle $\beta_{\lambda}$ given by

$$
m \lambda=d\left[\sin (\alpha)+\sin \left(\beta_{\lambda}\right)\right]
$$

where $m$ is the integer of propagating diffraction orders, being $-2 d<m \lambda<2 d$ and $d$ is generally represented as groove density $G=1 / d$, in lines/mm or grooves $/ \mathrm{mm}$.

Blazed diffraction gratings are optimized to achieve maximum grating efficiency at a specific $m$ and $\lambda$ (blaze $\lambda$ ), which is defined by the grooves shape, especially by $\theta_{B}$.

\section{A. Central Wavelength in Littrow Configuration}

The Mux/DeMux is designed for $\lambda_{S}$ and $\lambda_{L}$, and their required spatial separation, represented by $S=y_{L}+y_{S}$, see Fig. 1(a). This design can be done optimizing the ILs of specific chs, as ports closest to $P_{\lambda C}$ have a better coupling efficiency. This can be used in order to fulfill the power requirements of a specific WDM system. In our general case, there are two main objectives: 1) minima ILs and 2) chs uniform ILs or all chs ILs near to the minimum value. The system shown in Fig. 1 is bidirectional. Therefore, for simplicity, the design process is done only in the demultiplexing direction; see Fig. 1(a).

The diffraction scheme is done using a Littrow configuration for $\lambda_{C}$, in order to obtain a fiber-to-fiber coupling efficiency between $P_{\lambda C}$ and the extremes ports approximately equal. In a Littrow configuration, the diffracted beam $\left(\lambda_{C}\right)$ is back-reflected in the incident beam direction, $\beta_{\lambda C}=\alpha$, and from (1) this is achieved when

$$
\sin (\alpha)=m \lambda_{c} /(2 d) .
$$

The maximum diffraction efficiency at $\lambda_{C}$ (for optimum $I L$ ), $\alpha=\beta_{\lambda C}=\theta_{B}$ needs to be met. In a bidirectional system, it is possible to prevent that some of the reflected light returns to the central port including a tilt angle in the diffraction grating about the $y$-axis $(\varphi)$.

On the other hand, $\lambda_{S}$ and $\lambda_{L}$ have diffraction angles $\beta_{\lambda S}$ and $\beta_{\lambda L}$, respectively; see Fig. 1(a). These angles are measured from $\beta_{\lambda C}$ as $\beta_{S}$ and $\beta_{L}$ (see Fig. 3) and are given by

$$
\beta_{\{S, L\}}=\alpha-\sin ^{-1}\left[m \lambda_{\{S, L\}} / d-\sin (\alpha)\right] .
$$

The $c h s$ centered at $\lambda_{S}$ and $\lambda_{L}$ are spatially separated, in the $y$-axis direction, from the central point $C$, a distance given by

$$
y_{\{S, L\}}=f \tan \left(\beta_{\{S, L\}}\right) .
$$

$\lambda_{C}$ is a wavelength that, considering a specific diffraction grating (fixed $d$ and $m$ ), generates the same angular dispersion

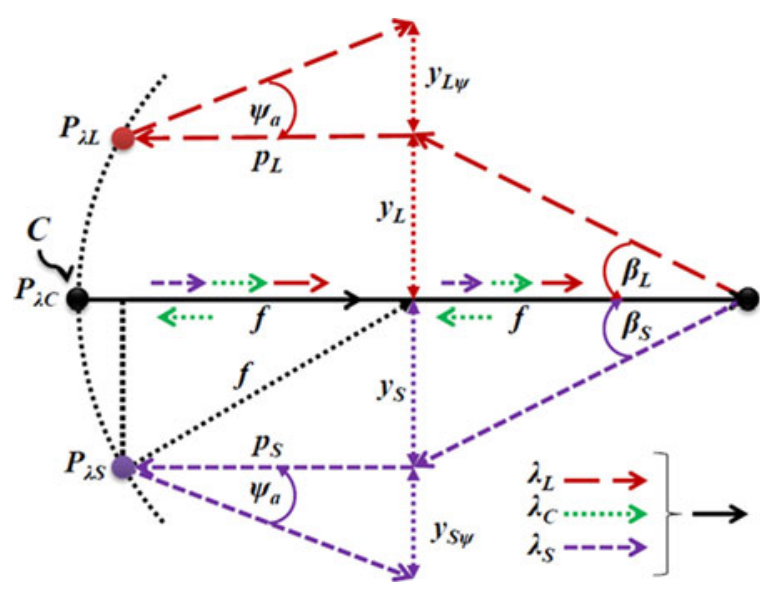

Fig. 3. Diameter of the lens required for the transmission of the extreme chs.

between $\lambda_{S}$ and $\lambda_{L}\left(\beta_{S}=\beta_{L}\right)$. Therefore, from (2) and (3), $\lambda_{C}$ must satisfy the following condition:

$$
\begin{aligned}
& \sin ^{-1}\left[\frac{m\left(2 \lambda_{S}-\lambda_{C}\right)}{2 d}\right]+\sin ^{-1}\left[\frac{m\left(2 \lambda_{L}-\lambda_{C}\right)}{2 d}\right] \\
& =2 \sin ^{-1}\left[\frac{m \lambda_{C}}{2 d}\right] .
\end{aligned}
$$

Fig. 2 shows $\lambda_{C}$ satisfying (5) and $\alpha=\beta_{\lambda C}$ from (2) versus the diffraction grating groove density, considering $\lambda_{S}=405$ $\mathrm{nm}$ and $\lambda_{L}=650 \mathrm{~nm}$. $\lambda_{C}$ can be approximated to $\lambda_{C}=\left(\lambda_{S}+\right.$ $\left.\lambda_{L}\right) / 2=527.5 \mathrm{~nm}$ for $G<1200$ lines/mm (see Fig. 2).

\section{B. Lens' $f$-number and Diameter}

SI-POF Muxes/DeMuxes require a higher spatial separation between the different chs than the GOs Muxes/DeMuxes, due to the larger SI-POF cladding diameter. A five $c h$ SI-POF Mux/DeMux requires $S=\left(\Delta y_{S}+\Delta y_{L}\right)>5 \mathrm{~mm}$. This value is 8 times greater than the required GOs $S$ in $125 \mu \mathrm{m}$ cladding diameter multimode fibers. As shown in (1) and (4), $S$ can be increased by increasing $G$ or $f$. The design criteria for selecting the optics (collimating/focusing lens), depending on $S, G$, and $f$, for the efficient transmission of all the chs is given as follows.

Beams in free space from SI-POFs have a diameter

$$
D_{F}=2 f \tan \left(\sin ^{-1} N A\right) \text {. }
$$

$N A$ range where the lens efficiently transmits the beam is represented as the $f$-number, defined as the ratio of $f$ to the diameter of the lens' entrance pupil, $D_{L}$, expressed as

$$
f / \#=f / D_{L} .
$$

Therefore, in order to collimate the beam that emerges from an SI-POF with $N A=0.48$ and diameter $D_{F}$, from (6), it is required an $f / \#<0.91$, considering that $D_{L}=D_{F}$ in (7).

The proposed diffraction scheme is symmetrical, if condition (5) is fulfilled. However, in practical cases, it is normal that $y_{S}+$ $y_{S \psi} \neq y_{L}+y_{L \psi}$, especially due to the variation of $f$ with the $\lambda$, or due to choosing a $\lambda_{C}$ not satisfying the symmetrical condition (5) to overcome the extreme $\operatorname{chs}\left(\lambda_{S}\right.$ and $\left.\lambda_{L}\right)$ differences on their 


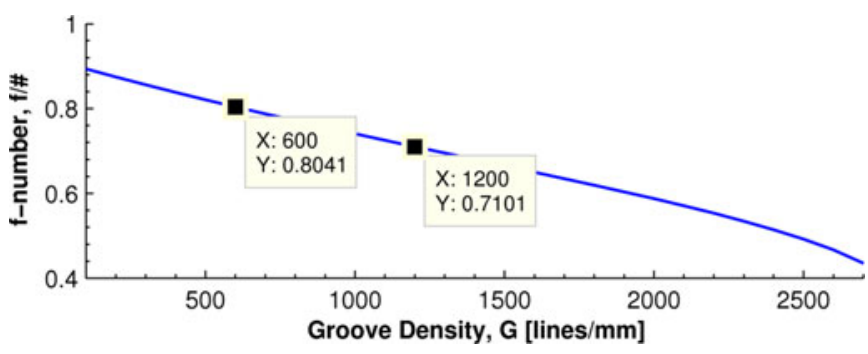

Fig. 4. $f$-number to transmit the resulting beams versus the groove density.

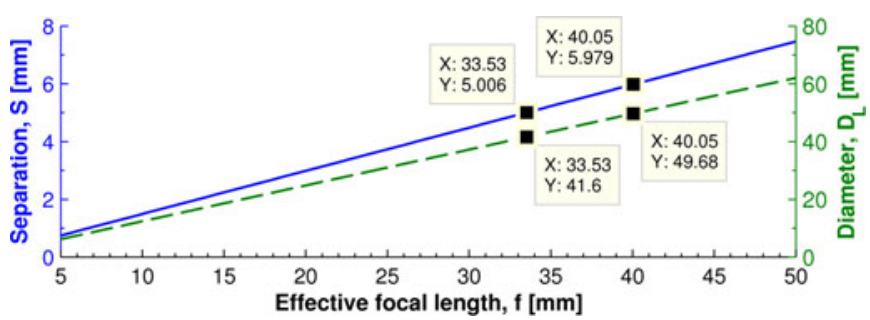

Fig. 5. Spatial separation (solid line, left vertical axis) and beam diameter (dashed line, right vertical axis) versus $f$. $G=600$ lines $/ \mathrm{mm}$.

diffraction efficiencies. The optics must be able to transmit the multiple beams (from the different input/output ports) over its entrance pupil, with total diameter $D_{L}$. From Fig. 3, the diameter must be greater than

$$
D_{L} \geq \max \left[y_{S}+y_{S \psi}, y_{L}+y_{L \psi}\right]
$$

where $y_{S \psi}=p_{S} \times \tan \left(\psi_{a}\right)$, with $p_{S}=\left(f^{2}-y_{S}^{2}\right)^{1 / 2}$ and $\psi_{a}=\sin ^{-1} N A$; and $y_{L \psi}=p_{L} \times \tan \left(\psi_{a}\right)$ with $p_{L}=\left(f^{2}-\right.$ $\left.y_{L}^{2}\right)^{1 / 2} \cdot p_{S}$ and $p_{L}$ can be approximated to $f$, if $f>>y_{S}$ and $f>>y_{L}$ (e.g., with $y_{S}$ and $y_{L}<4 \mathrm{~mm}$ and $f>20 \mathrm{~mm}$, the relative error of this approximation is less than $2 \%$ ). Therefore, the required optics must have

$$
\begin{aligned}
& f / \# \leq \min \left\{\left[\tan \left(\beta_{S}\right)+\tan \left(\psi_{a}\right)\right]^{-1},\right. \\
& \left.\left[\tan \left(\beta_{L}\right)+\tan \left(\psi_{a}\right)\right]^{-1}\right\} .
\end{aligned}
$$

Then, $f / \#$ is almost independent of $f$ if $f>>y_{S}$ and $f>>$ $y_{L}$. Fig. 4 shows $f / \#$ versus $G$ from (9) with $\lambda_{S}=405 \mathrm{~nm}$, $\lambda_{L}=650 \mathrm{~nm}$ and SI-POFs with $N A=0.48$, for $m=1$. The required $f / \#$ decreases as the grating $G$ increases. The $f / \#$ required for both $G=600$ lines/mm $(f / \#<0.8)$ and $G=1200$ lines/mm $(f / \#<0.71)$ is satisfied using aspheric lenses.

\section{Spatial Separation Between Channels}

Channels' spatial separation depends on $G$ and $f$. Higher $G$ values require more complex optics and can reduce the grating efficiency, due to the manufacturing tolerances. But, large $f$ values results in bulky systems. The tradeoff between $G$ and $f$ is analyzed in the following example. The objective is to separate more than five SI-POF $c h s$, placed in a spectrum from $\lambda_{S}=405$ $\mathrm{nm}$ to $\lambda_{L}=650 \mathrm{~nm}$, therefore, $S=y_{S}+y_{L}>5 \mathrm{~mm}$.

In this example, we consider a diffraction grating with $G=$ 600 lines/mm. Fig. 5 shows the resulting $S$ and the entrance pupil diameter, $D_{L}$, of the lens required as a function of $f$,

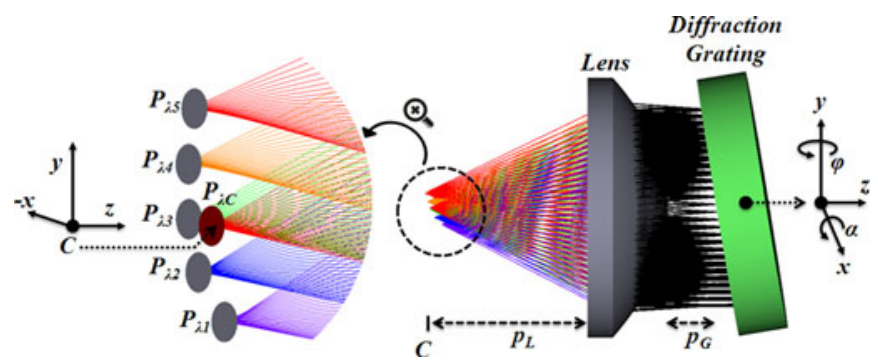

Fig. 6. Layout of the five channel SI-POF Mux/DeMux.

from (4), (5), and (8). It can be shown that $S>5 \mathrm{~mm}$ can be obtained using a lens with $f>33.5 \mathrm{~mm}$ and $D_{L}>41.6$ $\mathrm{mm}(f / \#<0.8$, see Fig. 4 for $G=600$ lines/mm). If $G$ increases up to 1200 lines/mm, $S$ doubles but the optics' $f$ / \# must be $<0.71$

\section{Five channels SI-POF MUX/DEMUX}

Equations previously analyzed are based on ideal elements. An optimized design, based on the characteristics of real optical elements, is now reported. It is based on the example analyzed in Section III-C; see Fig. 6. The input/output ports are named $P_{\lambda n}$, with $n=1,2 \ldots, 5$, and their $c h s$ cover the spectral range of 405-650 nm: $\lambda_{1}=\lambda_{S}=405 \mathrm{~nm}, \lambda_{2}=466.25 \mathrm{~nm}, \lambda_{3}=$ $527.5 \mathrm{~nm}, \lambda_{4}=588.75 \mathrm{~nm}$, and $\lambda_{5}=\lambda_{L}=650 \mathrm{~nm}$; which correspond to chs: $1,4,7,10$, and 13 of a previous SI-POF visible WDM grid proposal [12]. A distance greater than $1 \mathrm{~mm}$ between consecutive ports is fulfilled using an aspheric lens with $f=40 \mathrm{~mm}$ and $D_{L}=50 \mathrm{~mm}$, see Section III-C. In this case, the spatial separation between the extreme chs is about $6 \mathrm{~mm}$; see Fig. 5. The considered diffraction grating has an area of $50 \mathrm{~mm} \times 50 \mathrm{~mm}, G=600$ lines $/ \mathrm{mm}, \theta_{B}=8.62$ and diffraction efficiency between $56 \%$ and $68 \%$ (for nonpolarized light) in the range of 400-650 nm [25].

Fig. 6 shows how the common port, $P_{\lambda C}$, is placed at the system center, $C$ point ( $y=0, x=0)$. Ports are separated $1 \mathrm{~mm}$ from $C$ in the $x$-axis direction; this separation is controlled by the diffraction grating tilt angle about the $y$-axis $(\varphi)$, for a fixed $f$ value. The distance of each port from $C$ in the $y$-axis direction is defined by $\Delta y_{n}$. The distance of each port from $C$ in the $z$-axis is defined by $\Delta z_{n}$. Finally, $p$ and $p_{G}$ are the distances from $C$ to the Lens surface 1, and from the Lens surface 2 to the grating surface, respectively, in the $z$-axis direction $(x=$ 0 and $y=0$ ). The design is done using a ray tracing optical design software. The main target is to optimize the fiber-to-fiber coupling efficiency $\left(\eta_{C n}\right)$ from the port $P_{\lambda C}$ to the different $c h s$ $\left(P_{\lambda n}\right)$ ports. It is also targeted uniform ILs of the extreme $c h s$ ( $P_{\lambda 1}$ and $P_{\lambda 5}$ ), by a proper selection of $\lambda_{C}$. Table II summarizes the characteristics of the proposed design, based on Fig. 6.

The coupling efficiency is calculated considering a circular object with $1 \mathrm{~mm}$ of diameter (central port, $P_{\lambda C}$ ), with $N A$ of 0.5 (worst case) and a uniform radiation profile (worst case); see Fig. 7. This means that each point in the SI-POF surface is radiating with the same intensity and with a $N A$ of $0.5 . \eta_{C n}$ is calculated from the rays that reach an image of $1 \mathrm{~mm}$ of diameter with $N A<0.5$ using a geometric image analysis [26]. 
TABLE II

SPECIFICATIONS OF THE Five ChANNEL MuX/DeMuX, $G=600$ LinES/MM

\begin{tabular}{|c|c|c|c|c|c|c|}
\hline \multicolumn{7}{|c|}{ General Characteristics } \\
\hline \multicolumn{7}{|c|}{$\begin{array}{l}\text { Central wavelength, } \lambda_{C}=540 \mathrm{~nm} \text { (for } I L \text { of } P_{\lambda 1}=I L \text { of } P_{\lambda 5} \text { ) } \\
p=30.479 \mathrm{~mm}, p_{G}=15 \mathrm{~mm}, \alpha=\beta_{\lambda C}=-9.327^{\circ}, \varphi=-0.727^{\circ}\end{array}$} \\
\hline $\begin{array}{l}\text { Channel/ } \\
\text { Port } P_{\lambda \mathrm{n}}\end{array}$ & $\lambda_{n}[\mathrm{~nm}]$ & $\Delta z_{n}[\mathrm{~mm}]$ & $\Delta y_{n}[\mathrm{~mm}]$ & $\eta_{C n}[\%]$ & $\begin{array}{c}\eta_{G n} \\
{[\%]}\end{array}$ & $\begin{array}{c}I L[\mathrm{~dB}] \\
(\text { Ex- } \\
\text { pected) } \\
\end{array}$ \\
\hline$\overline{P_{\lambda 1}}$ & 405.00 & 2.325 & -3.10 & 79 & 62 & 3.1 \\
\hline$P_{\lambda 2}$ & 466.25 & 0.984 & -1.75 & 88 & 67 & 2.3 \\
\hline$P_{\lambda 3}$ & 527.50 & 0.130 & -0.30 & 94 & 67 & 2.0 \\
\hline$P_{\lambda 4}$ & 588.75 & -0.434 & 1.18 & 93 & 63 & 2.3 \\
\hline$P_{\lambda 5}$ & 650.00 & -0.813 & 2.70 & 88 & 56 & 3.1 \\
\hline \multicolumn{7}{|c|}{ Spatial Separation } \\
\hline $\begin{array}{l}\mid \Delta y_{1}-\Delta \\
\Delta y_{5} \mid=1\end{array}$ & $\begin{array}{l}1.35 \\
=\mid \Delta y\end{array}$ & $\begin{array}{l}-\Delta y_{3} \mid \\
\Delta y_{1} \mid=5\end{array}$ & $1.45, \mid \Delta y_{3}$ & ${ }_{3}-\Delta y_{4} \mid=$ & & $\Delta y_{4}-$ \\
\hline
\end{tabular}

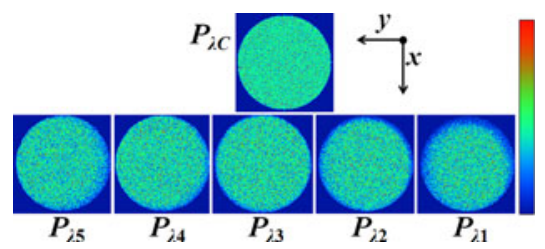

Fig. 7. Beam profiles of multimode fiber-to-fiber coupling efficiency calculations between $P_{\lambda C}$ and each input/output port.

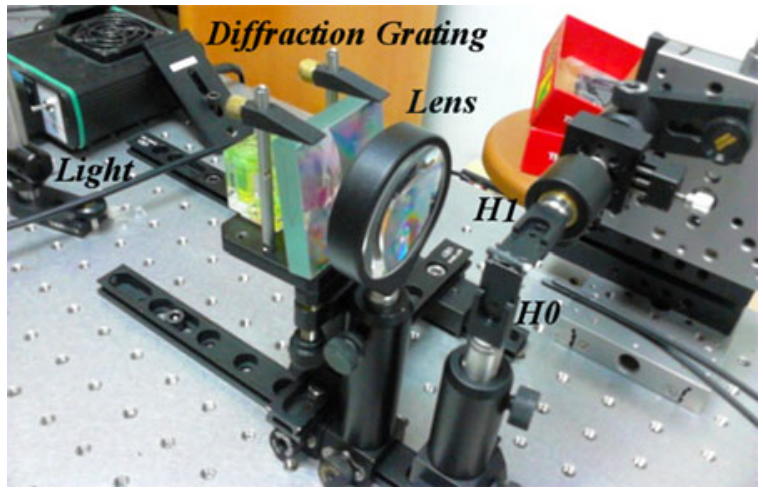

Fig. 8. Experimental setup for the characterization of the five $\operatorname{ch}$ Mux/DeMux: $H_{0}$ : holder of the common fiber, $H_{1}$ : input/output fiber holder.

Fig. 7 shows the resulting beam profile at the end face of each SIPOF input/output port and Table II shows the respective values of $\eta_{C n}$. Each $c h$ expected $I L$ is calculated with $\eta_{C n}$ and the approximated grating efficiency $\left(\eta_{G n}\right)$ taken from [25]. The spatial separation between adjacent $c h s$ is greater than $1.35 \mathrm{~mm}$, with $S=5.8 \mathrm{~mm}$ (see Table II). This value is in good agreement with values predicted on Fig. 5 .

\section{Experimental characterization}

The experimental setup is shown in Fig. 8. The distance from the fiber holders $\left(H_{0}\right.$ and $\left.H_{1}\right)$ to the rear surface of the grating is about $65 \mathrm{~mm}$. It has a common port, $P_{\lambda C}\left(H_{0}\right)$ and five input/output ports, $P_{\lambda n}\left(H_{1}\right)$, with $n=1,2 \ldots, 5$. Each input/output port has its own focusing distance $\left(p+\Delta z_{n}\right)$; see Table II. The transfer function characterization is done by

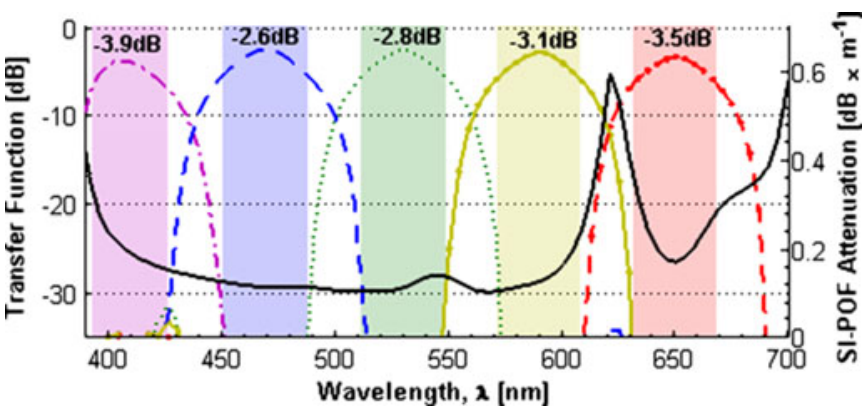

Fig. 9. Mux/DeMux transfer function (left vertical axis): $\lambda_{1}$ (dash-dot line), $\lambda_{2}$ (dashed line), $\lambda_{3}$ (dotted line), $\lambda_{4}$ (solid line with point markers), $\lambda_{5}$ (dashed line with point markers). SI-POF attenuation (solid line, right vertical axis).

moving the $H_{1}$ holder to the different $c h$ positions with a micrometric $x y z$ translation stage.

Spectral measurements are done using a Halogen light source and a high speed Spectrometer with spectral resolution of about $4 \mathrm{~nm}$ in the spectral range from 360 to $886 \mathrm{~nm}$, using the following procedure: 1) the light source is connected to the spectrometer using $3 \mathrm{~m}$ of SI-POF, with mode scramblers next to the source and to the spectrometer, this measurement represents the reference spectra $(0 \mathrm{~dB}$ reference), 2$)$ the $3 \mathrm{~m}$ of SI-POF is cut in half and each end is polished, 3) the section attached to the light source is connected to the common port holder $\left(H_{0}\right)$ and the section that is attached to the spectrometer is connected to the input/output port holder $\left(H_{1}\right)$, and 4) the holder $H_{1}$ is positioned at the different port locations, from Table II.

The transfer function of each input/output port in the spectral range from 390 to $700 \mathrm{~nm}$ is shown in Fig. 9. The $I L$ per $c h$ is less than $4 \mathrm{~dB}$. This value includes the $I L$ produced by two polished surfaces. Fresnel losses per PMMA-air interface is typically $\sim 4 \%$ (lens is AR coated: $350-700 \mathrm{~nm}$ ). The spectral bandwidth at $-25 \mathrm{~dB}$ is higher than $38 \mathrm{~nm}$. The $3 \mathrm{~dB}$ spectral bandwidths are represented by shaded areas on Fig. 9 . The $3 \mathrm{~dB}$ spectral bandwidth of each $c h$ is greater than $30 \mathrm{~nm}$. And the crosstalk attenuation (CTA) is greater than $30 \mathrm{~dB}$. The CTA is a measure of the part of the optical power at each $\lambda$ exiting from the port $P_{\lambda n}$ at wavelengths outside its $3 \mathrm{~dB}$ bandwidth. Measurements $40 \mathrm{~dB}$ below the reference signal are limited by the spectrometer sensitivity. The SI-POF fiber attenuation at 405 , $470,530,588$, and $650 \mathrm{~nm}$ is $0.21,0.11,0.11,0.12$, and $0.17 \mathrm{~dB} /$ $\mathrm{m}$, respectively. Table III shows the five $c h$ Mux/DeMux parameters. This table also presents an experimental and expected ILs comparison. It can be seen that there is a good agreement between them.

\section{Discussion}

The proposed diffraction scheme is used for designing an efficient and compact five $c h$ Mux/DeMux for visible WDM transmission over SI-POF technology. This Mux/DeMux is able to separate adjacent SI-POF $c h s$ a distance greater than $1.35 \mathrm{~mm}$, with a total separation between chs of $5.8 \mathrm{~mm}$. The spectral bandwidths at -3 and $-25 \mathrm{~dB}$ are greater than 30 and $38 \mathrm{~nm}$, 
TABLE III

Performance Summary of the Proposed Five Channel MuX/DeMuX

\begin{tabular}{|c|c|c|c|c|c|}
\hline \multirow[b]{2}{*}{$\begin{array}{l}\text { Channel/ } \\
\text { Port } P_{\lambda n}\end{array}$} & \multirow[t]{2}{*}{$\lambda[\mathrm{nm}]$} & \multicolumn{2}{|c|}{ Band-pass at: } & \multicolumn{2}{|c|}{ Insertion Losses } \\
\hline & & $-3 \mathrm{~dB}$ & $-25 \mathrm{~dB}$ & Experimental & $\begin{array}{c}\text { Experimental } \\
\text { - Expected }\end{array}$ \\
\hline$P_{\lambda 1}$ & 405 & $30 \mathrm{~nm}$ & $43 \mathrm{~nm}$ & $3.9 \mathrm{~dB}$ & $0.8 \mathrm{~dB}$ \\
\hline$P_{\lambda 2}$ & 470 & $35 \mathrm{~nm}$ & $43 \mathrm{~nm}$ & $2.6 \mathrm{~dB}$ & $0.3 \mathrm{~dB}$ \\
\hline$P_{\lambda 3}$ & 530 & $38 \mathrm{~nm}$ & $42 \mathrm{~nm}$ & $2.8 \mathrm{~dB}$ & $0.8 \mathrm{~dB}$ \\
\hline$P_{\lambda 4}$ & 588 & $37 \mathrm{~nm}$ & $41 \mathrm{~nm}$ & $3.1 \mathrm{~dB}$ & $0.8 \mathrm{~dB}$ \\
\hline$P_{\lambda 5}$ & 650 & $37 \mathrm{~nm}$ & $42 \mathrm{~nm}$ & $3.5 \mathrm{~dB}$ & $0.4 \mathrm{~dB}$ \\
\hline
\end{tabular}

Expected $I L s$ are given in Table II.

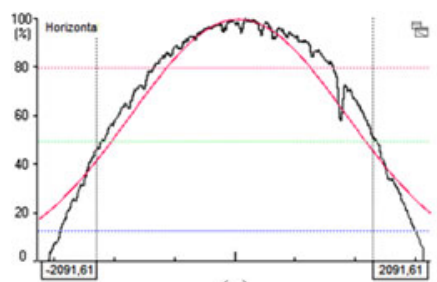

(a)

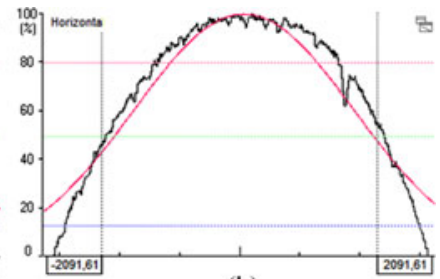

(b)
Fig. 10. Output beam profile measures: (a) without mode scramblers (FWHM $=4166.67 \mu \mathrm{m})$; (b) with mode scramblers (FWHM $=4234.99 \mu \mathrm{m})$.

respectively, in all the chs. The most important characteristics of the proposed Mux/DeMux are its chs low and uniform ILs.

These characteristics allow the implementation of efficient transmission links based on visible WDM over SI-POF, since the power penalty, produced by the low ILs, does not impose a limit to the real improvement of the link capacity. Some authors [9], [21] set the ILs per ch to $5 \mathrm{~dB}$ as a reasonable value, for a real increase in the link transmission capacity using SI-POF visible WDM. ILs of the Mux/DeMux are less than $4 \mathrm{~dB}$ and loss uniformity is $1.1 \mathrm{~dB}$.

Another aspect that must be highlighted is that none of the recently proposed SI-POF links based on visible WDM are bidirectional [5], [13]. This is due to the high ILs of the current Mux/DeMux proposals or due to their complexity and size. The proposed five chs Mux/DeMux allows the implementation of bidirectional links, made entirely with SI-POFs, including ILs $<8 \mathrm{~dB}$ ( $<4 \mathrm{~dB}$ in mux and $<4 \mathrm{~dB}$ in demux). It is important to note that a simple $4: 1$ SI-POF coupler have ILs up to $8 \mathrm{~dB}$ [7].

From Tables II and III, it is shown that there is a good agreement between the theoretical design and the experimental results. The differences are mainly due to the effects of Fresnel losses in each PMMA-air interface, to the imperfections in the POF surfaces and to the tolerances in the experimental setup.

The use of the mode scrambler at the POF input results in the same angular emission pattern independently of the chosen light source, which greatly improves the measurements realiability and repeatability [7]. Fig. 10 shows the measurements of the horizontal beam profile at $\sim 5 \mathrm{~mm}$ of $P_{\lambda C}$ when a blue LED source (IF E92) is launched on the input fiber, with and without mode scramblers. It can be shown that there is no reduction in the beam profile. On the other hand, Fig. 11 shows the characterization of the shortest and longest wavelength channels with

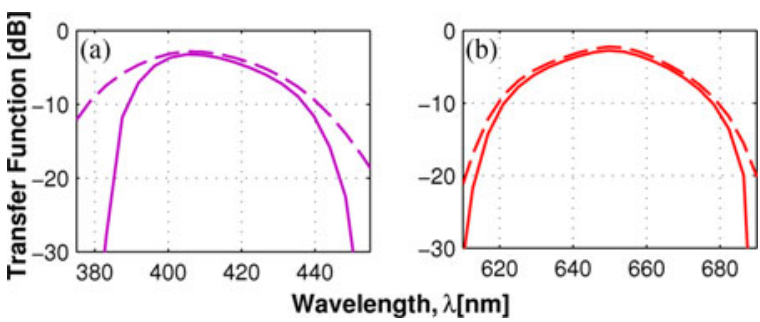

Fig. 11. Mux/DeMux characterization with (solid lines) and without (dashed lines) mode scramblers: (a) port $P \lambda 1=405 \mathrm{~nm}$, (b) port $P \lambda 5=650 \mathrm{~nm}$. ILs of the polished surfaces at the input/output ports are not included.
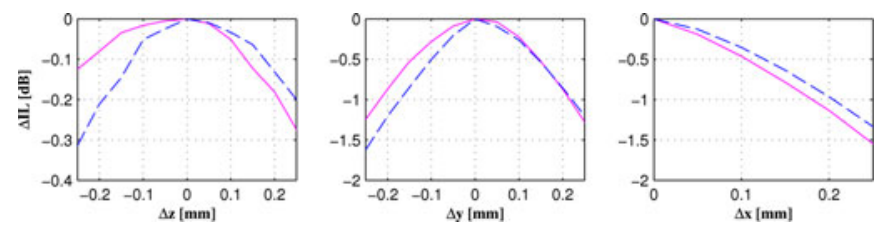

Fig. 12. Insertion losses variation $(\Delta I L)$ versus $z, y$ and $x$ positions, for channels centered at $405 \mathrm{~nm}$ (solid line) and at $650 \mathrm{~nm}$ (dashed line).

and without mode scramblers. It can be seen that lower insertion losses, about $-0.5 \mathrm{~dB}$ (at the central wavelength), are obtained when mode scramblers are not used. Therefore, the $I L$ measured using mode scramblers can be considered as a limit of the worst expected value.

\section{A. Tolerances and Ports' Central Wavelength Tuning}

The tolerances of the proposed Mux/DeMux are mainly related to the position tolerance of the output ports, since it is the most sensitive parameter. The position of the output ports (holder $H_{1}$ in Fig. 8) is controlled with a micrometric $x y z$ translation stage. The other distances in the system are adjusted with much less precision tools. The ILs of the extremes ports are the most sensitive to the variations in their optimized positions. Fig. 12 shows the experimental characterization of the ILs variation $(\Delta I L)$ of the ports $P_{\lambda 1}$ and $P_{\lambda 5}$ against the variations in the $z, y$, and $x$ direction from their optimized positions $\left(\Delta z_{n}+\Delta z, \Delta y_{n}+\Delta y, \Delta x_{n}+\Delta x\right)$; see Table II. It can be seen that the variations in the $z$ direction of up to $\pm 0.25 \mathrm{~mm}$ increase the ILs in less than $0.3 \mathrm{~dB}$. On the other hand, the variations in the $y$ and $x$ direction have a higher impact in the $\Delta I L$. In order to maintain the $\Delta I L$ of both $c h s$ below $-0.5 \mathrm{~dB}$, the variations in the $y$ and $x$ directions must be less than \pm 0.1 and $0.1 \mathrm{~mm}$, respectively.

Ports position shifts in the $y$ direction has an almost linear effect in their central $\lambda$ shifts, $\Delta \lambda$. The Mux/DeMux has a $\Delta \lambda[\mathrm{nm}]$ in all the $c h s$ of about $43.48 \times \Delta y[\mathrm{~mm}]$.

The separation between adjacent chs is greater than $1.35 \mathrm{~mm}$. Therefore, in a final application, the central $\lambda$ of each port shown in Fig. 9 can be tuned up to $\Delta \lambda=15.22 \mathrm{~nm}(\Delta y=0.3 \mathrm{~mm})$, in order to accommodate specific chs. Obviously, this fact reduces the band-pass bandwidths. However, even if the pass bandwidths values reported in Table II are reduced by $15 \mathrm{~nm}$, they still allow the use of laser sources, with little or no interference [5]. 


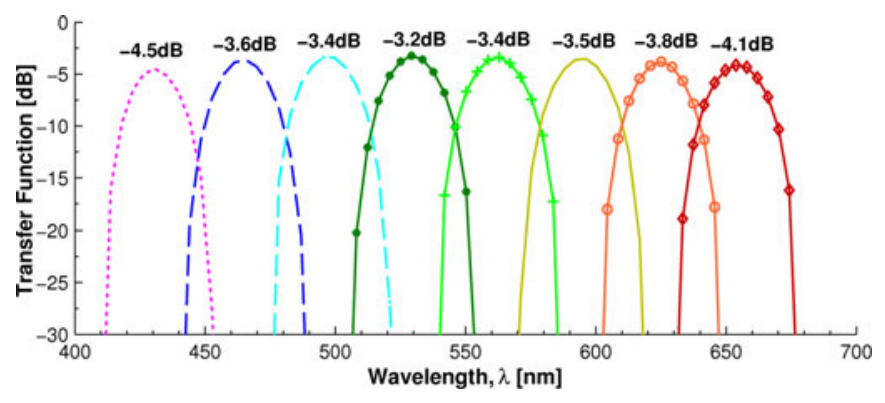

Fig. 13. Transfer function of an eight channel Mux/DeMux. The chs' central $\lambda$ are: $\lambda_{1}$ (dotted line), $\lambda_{2}$ (dashed line), $\lambda_{3}$ (dashed line with dot markers), $\lambda_{4}$ (solid line with $d o t$ markers), $\lambda_{5}$ (solid line with $\times$ markers), $\lambda_{6}$ (solid line), $\lambda_{7}$ (solid line with circle markers), and $\lambda_{8}$ (solid line with diamond markers).

\section{B. Scalability: Eight Channels Mux/DeMux}

It is possible to increase the spatial separation between the extreme chs to $S \approx 9 \mathrm{~mm}$ using a diffraction grating with $G$ $=1200$ lines $/ \mathrm{mm}$ and a lens with $f=35 \mathrm{~mm}$ and $D_{L} \geq 45$ $\mathrm{mm}(f / \#<0.71$; see Fig. 4). This configuration is also experimentally tested. However, due to the low performance of the available diffraction grating at $405 \mathrm{~nm}$, the design is optimized to accommodate eight $c h s$ in the range from 430 to $655 \mathrm{~nm}$. The experimental transfer function of each $c h$ is shown in Fig. 13. In this case, $\lambda_{1}=430 \mathrm{~nm}, \lambda_{2}=465 \mathrm{~nm}, \lambda_{3}=497 \mathrm{~nm}, \lambda_{4}=530$ $\mathrm{nm}, \lambda_{5}=562 \mathrm{~nm}, \lambda_{6}=595 \mathrm{~nm}, \lambda_{7}=625 \mathrm{~nm}$, and $\lambda_{8}=655$ $\mathrm{nm}$. The separation between consecutive chs is greater than $1.23 \mathrm{~mm}$, with total separation of $S=8.84 \mathrm{~mm}$ The $\Delta \lambda[\mathrm{nm}]$ in all the $c h s$ is $\approx 25.6 \times \Delta y[\mathrm{~mm}]$ and $I L s<4.5 \mathrm{~dB}$ with uniformity of $1.3 \mathrm{~dB}$. The size is reduced to about $57 \mathrm{~mm}$ of length by $50 \mathrm{~mm}$ of height. The spectral band-pass bandwidth at $-3 \mathrm{~dB}$ of all the chs is greater than $20 \mathrm{~nm}$, and their spectral band-pass bandwidths at $-25 \mathrm{~dB}$ are between 12 and $21 \mathrm{~nm}(16 \mathrm{~nm}$ at $P_{\lambda 1}, 17 \mathrm{~nm}$ at $P_{\lambda 2}, 17 \mathrm{~nm}$ at $P_{\lambda 3}, 21 \mathrm{~nm}$ at $P_{\lambda 4}, 21 \mathrm{~nm}$ at $P_{\lambda 5}$, $17 \mathrm{~nm}$ at $P_{\lambda 6}, 12 \mathrm{~nm}$ at $P_{\lambda 7}$, and $18 \mathrm{~nm}$ at $P_{\lambda 8}$ ). In comparison with the six ch Mux/DeMux reported in [5], this proposal is more compact and has half its ILs (having an improvement of up to $5 \mathrm{~dB}$ ).

\section{Size Reduction and Applicability}

It is possible to spatially separate five chs a total distance of about $6 \mathrm{~mm}$ using a diffraction grating with $G=1200$ lines $/ \mathrm{mm}$, and a lens with $f=20 \mathrm{~mm}$ and diameter of $D_{L}=25 \mathrm{~mm}$. The lens requirement can be fulfilled using an aspheric lens $(f / \#<0.7$; see Fig. 4). In this case, the Mux/DeMux size can be reduced to less than $37 \mathrm{~mm}$ in length and $30 \mathrm{~mm}$ in height, which represents a reduction factor of 1.75 in length and 2.2 in height.

Apart from visible WDM SI-POF links, these low insertion losses Mux/DeMux can also be used in intensity based fiberoptic sensing configurations using WDM as the self-referencing principle [27].

\section{Conclusion}

A five $c h$ Mux/DeMux for visible WDM transmission over SI-POF has been designed and experimentally tested. It is based on a reflective blazed diffraction grating and an aspherical lens. It has a length of $\sim 65 \mathrm{~mm}$ and height of $\sim 55 \mathrm{~mm}$. The experimental $I L$ and $C T A$ are found to be less than $4 \mathrm{~dB}$ and higher than $30 \mathrm{~dB}$, respectively, and the spectral band-pass bandwidths at -3 and $-25 \mathrm{~dB}$ in all the chs are greater than 30 and $38 \mathrm{~nm}$, respectively. Tolerance analysis shows that extremes ports $I L$ increments around $0.3-0.5 \mathrm{~dB}$ are expected for ports' positions shifts of up to $\pm 0.25, \pm 0.1$, and $+0.1 \mathrm{~mm}$ in the $z, y$, and $x$ directions, respectively. This analysis also shows that the central wavelength of each port can be tuned up to $15 \mathrm{~nm}$, in order to accommodate specific chs. The Mux/DeMux design can be extended to eight chs keeping the ILs $<4.5 \mathrm{~dB}$ and $C T A>30 \mathrm{~dB}$ in a device with length of $\sim 57 \mathrm{~mm}$ and height of $\sim 50 \mathrm{~mm}$. In this case, spectral band-pass bandwidth at $-25 \mathrm{~dB}$ is $12-21 \mathrm{~nm}$. Theoretical expectations are in good agreement with experimental results. This theoretical analysis can be used to design a five ch Mux/DeMux with a size reduction factor of 1.75 in length and of 2.2 in height, versus the five $c h$ design reported.

The Muxes/DeMuxes presented in this paper have the best characteristics, in terms of performance, number of chs and size, reported in experimental systems. These devices allow the implementation of visible WDM links over SI-POF with a low power penalty compared to single $c h$ systems.

\section{Acknowledgement}

The authors gratefully acknowledge discussions with Prof. K. Heggarty.

\section{References}

[1] E. (Yuxin) Dai. (2014, Oct.). Home Networking bandwidth growth needs and POF. Presented at IEEE 802.3 GEPOF Study Group Meeting. [Online]. Available: http://www.ieee802.org/3/GEPOFSG/email/pdfcnr2Yo_ YaJ.pdf

[2] Y. Shi, E. Tangdiongga, A. M. J. Koonen, A. Bluschke, P. Rietzsch, J. Montalvo, M. De Laat, G. Van Den Hoven, and B. Huiszoon, "Plasticoptical-fiber-based in-home optical networks," IEEE Commun. Mag., vol. 52, no. 6, pp. 186-193, Jun. 2014.

[3] I. Mollers, D. Jager, R. Gaudino, A. Nocivelli, H. Kragl, O. Ziemann, N. Weber, T. Koonen, C. Lezzi, A. Bluschke, and S. Randel, "Plastic optical fiber technology for reliable home networking: Overview and results of the EU project pof-all," IEEE Commun. Mag., vol. 47, no. 8, pp. 58-68, Aug. 2009

[4] M. Rayon Co. (2014, May). Plastic Optical Fiber (POF) technology for Automotive, Home network systems. Presented at IEEE 802.3 GEPOF Study Group meeting. [Online]. Available: http://www.ieee802. org/3/GEPOFSG/public/May_2014/Tsukamoto_GEPOF_01_0514.pdf

[5] R. Kruglov, J. Vinogradov, S. Loquai et al., "21.4 Gb/s Discrete Multitone Transmission over 50-m SI-POF employing 6-channel WDM," in Proc. Opt. Fiber Conf., Mar. 2014, pp. 1-3.

[6] C. M. Okonkwo, E. Tangdiongga, H. Yang, D. Visani, S. Loquai, R. Kruglov, B. Charbonnier, M. Ouzzif, I. Greiss, O. Ziemann, R. Gaudino, and A. M. J. Koonen, "Recent results from the EU POF-PLUS project: multi-Gigabit transmission over $1 \mathrm{~mm}$ core diameter plastic optical fibers," J. Lightw. Technol., vol. 29, no. 2, pp. 186-193, Jan. 2011.

[7] J. K. Olaf Ziemann, P. E. Zamzow, and Werner Daum, POF Handbook: Optical Short Range Transmission Systems, 2nd ed. New York, NY, USA: Springer, 2008.

[8] J. L. Wei, L. Geng, D. G. Cunningham et al., "Gigabit NRZ, CAP and optical OFDM systems over POF links using LEDs," Opt. Exp., vol. 20, pp. 22284-22289, 2012.

[9] O. Ziemann and L. Bartkiv, "POF-WDM, the Truth," in Proc. Int. Conf. Plastic Opt. Fiber, Bilbao, Spain, 2011, pp. 525-530.

[10] L. Geng, J. L. Wei, R. V. Penty, and I. H. White, "3 Gbit/s LED-based step index plastic optical fiber link using multilevel pulse amplitude 
modulation," presented at the Optical Fiber Communication Conf. Expo./Nat. Fiber Optic Engineers Conf., Anaheim, CA, USA, Paper OTh4A.1.

[11] O. Ciordia, C. Esteban, C. Pardo, and R. Pérez de Aranda, "Commercial silicon for gigabit communication over SI-POF," in Proc. Int. Conf. Plastic Opt. Fiber, Buzios, Brazil, 2013, pp. 109-116.

[12] M. Joncic, M. Haupt, and U. H. P. Fischer, "Standardization proposal for spectral grid for vis WDM applications over SI-POF," in Proc. Int. Conf. Plastic Opt. Fiber, Atlanta, GA, USA, 2012, pp. 351-355.

[13] M. Joncic, R. Kruglov, M. Haupt, R. Caspary, J. Vinogradov, and U. H. P Fischer, "Four-channel WDM transmission over 50-m SI-POF at 14.77 Gb/s using DMT modulation," IEEE Photon. Technol. Lett., vol. 26, no. 13, pp. 1328-1331, Jul. 2014.

[14] Y. Shi, C. Okonkwo, D. Visani, E. Tangdiongga, and T. Koonen "Distribution of broadband services over 1-mm core diameter plastic optical fiber for point-to-multipoint in-home networks," J. Lightw. Technol., vol. 31, no. 6, pp. 874-881, Mar. 2013.

[15] T. Fukushima, and T. Sakamoto, "A $7 \times 6$ optical fiber grating demultiplexer-multiposition switch for 0.64-0.88 $\mu \mathrm{m}$ band," J. Lightw. Technol., vol. 14, no. 5, pp. 867-872, May 1996.

[16] W. J. Tomlinson III, "Wavelength division multiplexer," Bell Telephone Laboratories Incorporated, U.S. Patent 4111 524, Sept. 5, 1978.

[17] A. A. M. Kok, S. Musa, A. Borreman et al., "Completely multimode arrayed waveguide grating-based wavelength demultiplexer," in Proc. EUROCON, vol. 2, Sept. 2003, pp. 422-426.

[18] H. P. A. van den Boom, W. Li, P. K. van Bennekom, I. T. Monroy, and G.D. Khoe, "High-capacity transmission over polymer optical fiber," IEEE J. Sel. Topics Quantum Electron., vol. 7, no. 3, pp. 461-470, May/Jun. 2001.
[19] S. Junger, W. Tschekalinskij, and N. Weber, "POF WDM transmission system for multimedia data," in Proc. Int. Conf. Plastic Opt. Fiber, Tokyo, Japan, 2002, pp. 69-71.

[20] D. Lutz, M. Haupt, and U. H. P. Fischer, "Demultiplexer for WDM over POF in prism-spectrometer configuration," in Proc. Int. Students Young Scientists Workshop Photon. Microsyst., Jun. 2008, pp. 43-46.

[21] L. V. Bartkiv, Y. V. Bobitski, and H. Poisel, "Optical demultiplexer using a holographic concave grating for POF-WDM systems," Optica Applicata, vol. 35, no. 1, pp. 59-66, 2005.

[22] S. Höll, M. Haupt, and U. H. P. Fischer, "Design and development of an injection-molded demultiplexer for optical communication systems in the visible range," Appl. Opt., vol. 52, no. 18, pp. 4103-4110, Jun. 2013

[23] M. Haupt, and U. H. P. Fischer, "Multi-colored WDM over POF system for Triple-Play," in Proc. SPIE, vol. 6992, pp. 699213-1-699215-10, May 03, 2008.

[24] P.J. Pinzón, K. Heggarty, C. Vázquez, and I. Pérez, "Diffraction gratingbased multiplexers for SI-POF networks," in Proc. Int. Conf. Plastic Opt. Fiber, Buzios, Brazil, 2013, pp. 36-41.

[25] Thorlabs. (2015, Mar.). GR50-0605 - Ruled Reflective Diffraction Grating. [Online]. Available: https://www.thorlabs.de/images/ tabImages/500_600_Ruled_Grating_Efficiency_Graph_780.gif

[26] Zemax. (2015, May). How to Model Coupling Into a Multi-Mode Fiber. [Online]. Available: https://www.zemax.com/support/resourcecenter/knowledgebase/how-to-model-coupling-into-a-multi-mode-fiber

[27] A. Tapetado, P. J. Pinzón, J. Zubia, and C. Vázquez, "Polymer optical fiber temperature sensor with dual-wavelength compensation of power fluctuations," IEEE J. Lightw. Technol., vol. 33, no. 13, pp. 2716-2764, 2015. 